\title{
Metastatic Lymph Node Ratio: A Promising Parameter and Its Relation with Patient Related Factors in Cases of Primary Organ Malignancy
}

\author{
Jansari Trupti Rajeshbhai ${ }^{1}$, Patel Jigna Prakashbhai ${ }^{2 *}$ \\ ${ }^{1}$ Affiliation - Assistant professor, Sumandeep Vidyapeeth, Piparia, Vadodara Address - C-10, Rupali Society, Sussen - Tarsali road, Opp. Motinagar - \\ 2, Vadodara - 390009 Gujarat, India \\ ${ }^{2}$ Assistant professor, Sumandeep Vidyapeeth, Piparia, Vadodara Address - 11- gopivallabh soc. Near Amin party plot, Gotri, Vadodara - 390021 \\ Gujarat, India
}

\author{
DOI: $10.36348 /$ sjpm.2019.v04i11.003 \\ | Received: 01.11.2019| Accepted: 08.11.2019| Published: 12.11.2019 \\ *Corresponding author: Patel Jigna Prakashbhai
}

Abstract

Background: Metastatic lymph node ratio (MLR) is defined as the ratio of number of positive lymph nodes (LNs) or LNM to total nodes harvested (LNH). Recent studies have suggested that MLR is more accurate in predicting overall survival and recurrence-free survival rate compared to the number of positive nodes alone. Materials and methods: Total 58 cases of primary organ malignancy were evaluated from June 2018 to June 2019 including Head and neck (HN), Breast, Gastrointestinal tract (GIT) and Genitourinary tract (GUT) malignancy. Age, gender, LNH, LNM, MLR, tumor stage and tumor grade were accessed for each of the cases. Results: Out of total 58, there were 12, 33, 11 and 2 cases each of HN, Breast, GIT and GUT malignancy respectively. M:F ratio was 1:2.2. Maximum cases (39.6\%) were seen in the age group of 35-45 years. 186 out of 530 LNs showed metastasis. Mean MLR for lymph node-positive cases were 0.3. Majority of HN malignancy cases (41.6\%) were found in T1/T2 stage, breast malignancy cases (42.4\%) in T2 stage, GIT malignancy cases $(63.6 \%)$ in T3 stage, $50 \%$ of GUT malignancy cases in T3 and 50\% were in T4 stage. Maximum well-differentiated $(10,83.3 \%)$ cases belonged to $\mathrm{HN}$, moderately $(15,45.4 \%)$ and poorly differentiated $(18,54.5 \%)$ to breast respectively. We found significant association of MLR with tumor stage and tumor grade. (p-value < 0.001$)$ Conclusion: The routine inclusion of MLR in tumor reporting by pathologists may become a prognostic aid for clinicians along with TNM staging system.

Keywords: Metastatic lymphnode ratio, primary organ malignancy, lymph node metastasis. Copyright @ 2019: This is an open-access article distributed under the terms of the Creative Commons Attribution license which permits unrestricted
use, distribution, and reproduction in any medium for non-commercial use (NonCommercial, or CC-BY-NC) provided the original author and sources
are credited.

\section{INTRODUCTION}

The status of regional lymph node metastasis (LNM) has been widely considered as an important prognostic factor to plan subsequent management of patients with primary organ malignancy [1]. Lymph nodes (LNs) draining a tumor may enlarge due to metastasis (LNM) or reactive hyperplasia [2]. One of the most commonly used prognostic factors is the tumor-node-metastasis (TNM) staging system [4]. Although LNM is a simple, convenient, and reliable method for precise staging, the identified number of metastatic LNs depends on the number of dissected LNs. A low number of examined LNs may lead to false understating and subsequent underestimation of cancer spread. This phenomenon is referred to as stage migration.
Metastatic lymph node ratio (MLR) is defined as the ratio of number of positive LNs or LNM to total nodes removed or harvested (LNH) and is used as an important prognostic factor. Recent studies have suggested that MLR is more accurate in predicting overall survival and recurrence-free survival rate compared to the number of positive nodes alone.

There is also disparity regarding the number of LNs to be resected [1]. The number of positive lymph nodes and the total number of dissected lymph nodes are affected by a lymph nodes dissection procedure and confirmed by pathological examination [4].

Some groups have recently proposed the metastatic lymph node ratio (MLR) as an alternative prognostic factor to supplement the limitations of the 
conventional $\mathrm{N}$ staging system, particularly when a limited number of LNs is obtained [3].

The routine inclusion of MLR in tumor reporting by pathologists may become a prognostic aid for clinicians alongside tumor stage and tumor grade [2].

\section{AIMS AND OBJECTIVES}

- To evaluate the prevalence of various primary organ malignancy and its metastasis.

- To evaluate the distribution of primary organ malignancy as per the age and gender of the patients.

- To evaluate total LNH, LNM, and MLR in resected specimens of primary organ malignancy.

- To evaluate the cases as per their MLR groups.

- To evaluate the relation of MLR with tumor stage and tumor grade.

\section{MATERIALS AND METHODS}

We carried out a retrospective observational study. In the current study, we included the cases of primary organ malignancies that came to the hospital over a period from June 2018 to June 2019. Total 58 resection specimens of primary organ malignancy with local LN clearance were included in this study.

Age, gender, LNH, LNM, MLR, tumor stage and tumor grade were accessed for each of the cases. All data were recorded in MS Excel sheet. These data were represented in tabulated as well as graphical formats. We calculated the $p-$ value as and when required and $\mathrm{p}$ - value of $<0.001$ was considered as significant. The statistical analysis was performed using MS Excel and SPSS software.

\section{RESULTS}

Total 58 cases of primary organ malignancies were studied in the present study. There were 12, 33, 11 and 2 cases each of Head and neck (HN), Breast, Gastrointestinal tract (GIT) and genitourinary tract (GUT) malignancy respectively [Table -1$]$. HN cases included buccal mucosa (5), tongue (5), and thyroid (2). GIT cases included colon (10) and stomach (1). GUT cases included penis (1) and kidney (1).

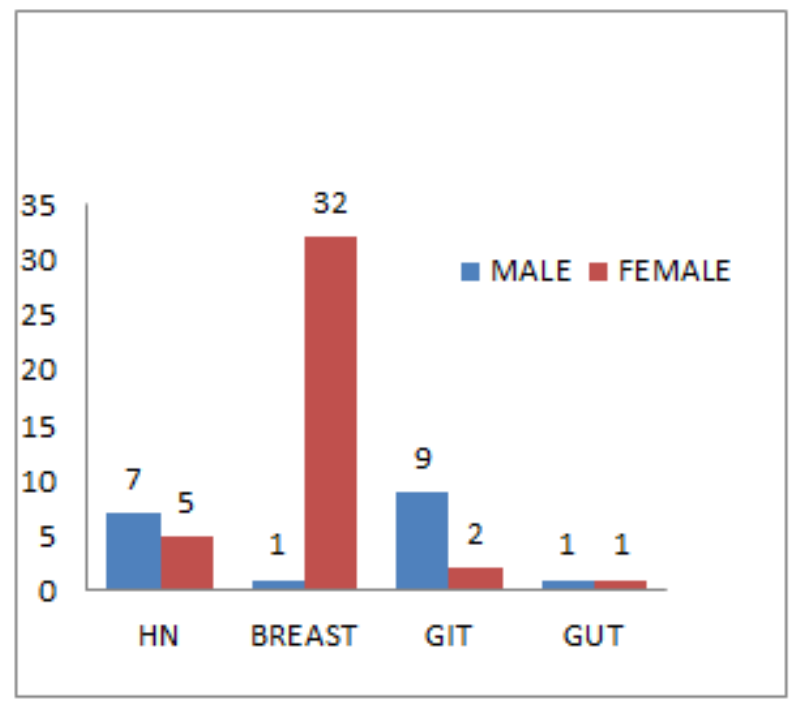

Fig-1: Distribution of cases as per the gender

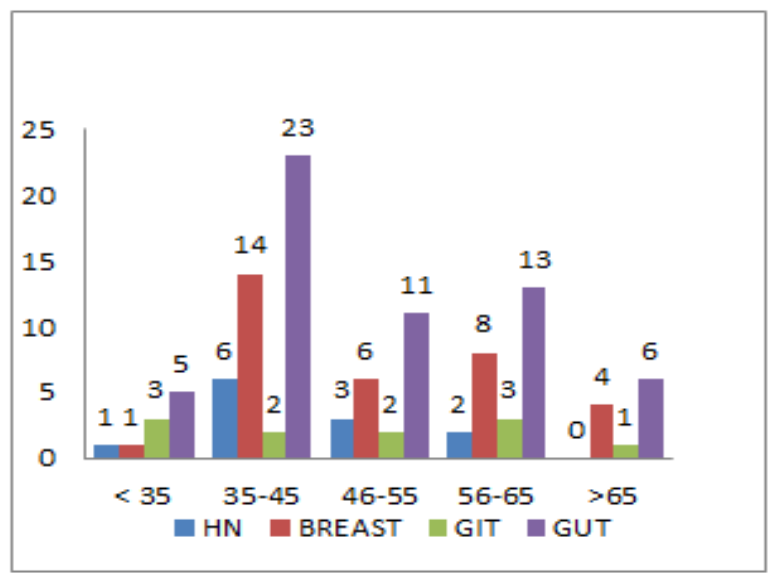

Fig-2: Distribution of cases as per the age group

There was a marked female preponderance (40) over males (18). [Figure - 1] Male to female ratio was 1:2.2. There was a marked female preponderance especially in cases of breast (96.9\%) with male preponderance in $\mathrm{HN}(58.3 \%)$ and GIT $(81.8 \%)$ cases. Maximum cases $(39.6 \%)$ of primary organ malignancies were seen in the age group of 35-45 years [Figure - 2].

LNH and LNM were evaluated for all the cases. Total 530 LNs were harvested from 58 cases. LNM were observed in 186 LNs (32 cases) [Table - 1].

Table-1: Distribution of cases as per primary organ malignancy, LNH and LNM

\begin{tabular}{|l|l|l|l|l|l|l|}
\hline $\begin{array}{l}\text { PRIMARY } \\
\text { ORGAN }\end{array}$ & $\begin{array}{l}\text { NO. OF } \\
\text { CASES }\end{array}$ & LNH & $\begin{array}{l}\text { MEAN } \\
\text { LNH }\end{array}$ & $\begin{array}{l}\text { CASES WITH } \\
\text { METASTASIS }\end{array}$ & LNM & $\begin{array}{l}\text { MEAN } \\
\text { LNM }\end{array}$ \\
\hline HN & 12 & 112 & 9.3 & 3 & 5 & 0.41 \\
\hline BREAST & 33 & 335 & 10.1 & 22 & 164 & 4.9 \\
\hline GIT & 11 & 76 & 6.9 & 4 & 11 & 1 \\
\hline GUT & 2 & 7 & 3.5 & 2 & 6 & 3 \\
\hline TOTAL & 58 & 530 & 29.8 & 31 & 186 & 9.31 \\
\hline
\end{tabular}


MLRO $\quad$ MLR1 MLR2 MLR3

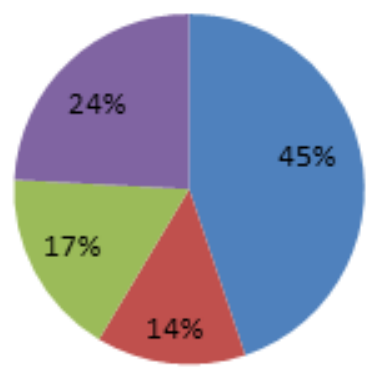

Fig-3: Distribution of cases as per the MLR groups

The ratio of metastatic $\mathrm{LN}$ to total number of resected $\mathrm{LN}$ was divided into four groups: MLR $0=0$, MLR $1=0-0.3$, MLR $2=0.3-0.6$ and MLR $3=0.6-1$ [Figure - 3]. The mean value of MLR for lymph nodepositive patients was 0.3 .

Mean MLR for HN was $0.03(0.0-0.18)$, for breast was $0.42(0.0-1.0)$, for GIT was $0.13(0.0-0.72)$, and for GUT was 0.83 (0.6-1.0). There were 7, 21, 22, and 8 cases, respectively, in tumor stage T1, T2, T3, and T4. [Table - 2]

Table-2: Distribution of cases as per the tumor stage

\begin{tabular}{|c|c|c|c|c|c|}
\hline PRIMARY ORGAN & T1 & T2 & T3 & T4 & TOTAL \\
\hline HN & 5 & 5 & 1 & 1 & 12 \\
\hline BREAST & 1 & 14 & 13 & 5 & 33 \\
\hline GIT & 1 & 2 & 7 & 1 & 11 \\
\hline GUT & 0 & 0 & 1 & 1 & 2 \\
\hline TOTAL & 7 & 21 & 22 & 8 & 58 \\
\hline
\end{tabular}

Majority of HN malignancy cases $(41.6 \%)$ were found in $\mathrm{T} 1 / \mathrm{T} 2$ stage and $83.3 \%$ were well differentiated. Majority of breast malignancy cases $(42.4 \%)$ were found in T2 stage and $54.5 \%$ poorly differentiated. Majority of GIT malignancy cases $(63.6 \%)$ were found in T3 stage and $54.5 \%$ were moderately differentiated. $50 \%$ of GUT cases were in $\mathrm{T} 3$ and $50 \%$ were in T4 stage and $50 \%$ was well differentiated and $50 \%$ were poorly differentiated. Distribution as per tumor grade showed well differentiated (14), moderately differentiated (22), and poorly differentiated (22) cases [Table - 3]. Maximum well differentiated $(10,83.3 \%)$ cases belonged to $\mathrm{HN}$, while maximum moderately differentiated $(15,45.4 \%)$ and poorly differentiated $(18,54.5 \%)$ to breast respectively. Maximum total cases in MLR3 group were poorly differentiated.
Table-3: Distribution of cases as per the tumor grade

\begin{tabular}{|c|c|c|c|c|}
\hline PRIMARY ORGAN & WD & MD & PD & TOTAL \\
\hline HN & 10 & 1 & 1 & 12 \\
\hline BREAST & 0 & 15 & 18 & 33 \\
\hline GIT & 3 & 6 & 2 & 11 \\
\hline GUT & 1 & 0 & 1 & 2 \\
\hline TOTAL & 14 & 22 & 22 & 58 \\
\hline
\end{tabular}

We evaluated the relation of MLR with tumor stage and tumor grade [Table $-4,5]$. There was significant association between MLR with tumor stage and tumor grade ( $\mathrm{p}$-value $<0.001)$.

Table-4: Relation between tumor stage and MLR

\begin{tabular}{|c|c|c|c|c|}
\hline $\begin{array}{c}\text { TUMOR } \\
\text { STAGE }\end{array}$ & MLR1 & MLR2 & MLR3 & TOTAL \\
\hline T1 & 1 & 0 & 1 & 2 \\
\hline T2 & 3 & 7 & 4 & 14 \\
\hline T3 & 3 & 3 & 7 & 13 \\
\hline T4 & 1 & 0 & 2 & 3 \\
\hline
\end{tabular}

Table-5: Relation between tumor grade and MLR

\begin{tabular}{|c|c|c|c|c|}
\hline $\begin{array}{c}\text { TUMOR } \\
\text { GRADE }\end{array}$ & MLR1 & MLR2 & MLR3 & TOTAL \\
\hline WD & 3 & 0 & 1 & 4 \\
\hline MD & 3 & 3 & 5 & 11 \\
\hline PD & 2 & 7 & 8 & 17 \\
\hline
\end{tabular}

\section{DISCUSSION}

The present study evaluated the data regarding age, gender, LNH, LNM, MLR, tumor stage and tumor grade from primary organ malignancy cases resected with local nodal clearance. LNH, LNM and MLR were studied for correlation with tumor stage and tumor grade.

The prevalence of involvement of primary organ in our study was HN $(20.6 \%)$, Breast $(56.9 \%)$, GIT (18.9\%) and GUT (3.4\%), which was comparable to the study done by Shinde V et al. [2].

The peak age group of affected patients in our study was 35-45 years. There was marked female preponderance over males in our study. These findings of our study were comparable with the study done by various other studies [2]. Akagi et al. and Zeng et al. reported male predominance in colorectal and gastric carcinoma, as did Chen et al. in or opharyngeal malignancy $[4,6,7]$. The male predominance in GIT and $\mathrm{HN}$ malignancies may reflect lifestyle related behavior as alcoholism, processed junk food, smoking, and tobacco chewing.

The recommended LNH values are 10, 12, 12, and 40 in breast, GIT, GUT, and HN respectively. Shinde et al. found mean $\mathrm{LNH}$ values 23.7, 15.5, 6.6 and 9.9 in HN, Breast, GIT and GUT respectively which were similar to our study. Techniques to further improve the yield of LNH include meticulous dissection 
during grossing, intratumoral India ink injection during surgery, ex-vivo intra-arterial injection of methylene blue, and chemical fat clearance using acetone and alcohol. Shinde et al. found mean LNM values 0.6, 4.4, 1.4 and 0.9 in HN, Breast, GIT and GUT respectively which were similar to our study [2].

We categorized all cases into four groups as per the MLR grouping. Our results showed 44.8\%, $13.7 \%, 17.2 \%$ and $24.13 \%$ cases in MLR0, MLR1, MLR2 and MLR3 groups respectively. Mean MLR in our study was 0.3 . Both these findings were comparable with the study done by Saedi et al. [3]

In our study, Mean MLR for $\mathrm{HN}$ was 0.03 (range $0.0-0.18)$, for breast was $0.42(0.0-1.0)$ for GIT was $0.13(0.0-0.72)$, and for GUT was $0.83(0.6-1.0)$ and it was comparable to the study done by Shinde V et $a l$. They found Mean MLR for HN 0.06 (0.0-0.67), for breast 0.29 (range $0.0-1.0$ ), for GIT $0.26(0.0-1.0)$ and for GUT $0.1(0.0-0.56)$.D2. They found maximum cases of breast $(59.6 \%)$, GUT $(53.8 \%)$, and HFNT (45\%) belonged to T2 stage, while GIT (60.0\%) belonged to T3. Distribution as per tumor grade showed well-differentiated (22), moderately differentiated (68), and poorly differentiated (10) cases. Maximum well-differentiated cases belonged to HFNT (13, $59.0 \%$ ), while maximum moderately differentiated and poorly differentiated to breast $(38,55.8 \%)$ and $(7$, $70.0 \%$ ), respectively as in our study [2].

LNH, LNM and LNR cutoff values show organ wise variation, hence need standardization before their usage in cancer staging systems. [1] Serial recuts, use of immunohistochemistry, one step nucleic acid amplification, and polymerase chain reaction can further aid in detecting minute nodal metastases [2].

In present study, majority of $\mathrm{HN}$ malignancy cases $(41.6 \%)$ were found in T1/T2 stage and $83.3 \%$ were well differentiated. Majority of breast malignancy cases $(42.4 \%)$ were found in T2 stage and $54.5 \%$ poorly differentiated. Majority of GIT malignancy cases $(63.6 \%)$ were found in $\mathrm{T} 3$ stage and $54.5 \%$ were moderately differentiated. $50 \%$ of GUT cases were in $\mathrm{T} 3$ and $50 \%$ were in T4 stage and 50\% was well differentiated and $50 \%$ were poorly differentiated. Titipungul et al. found majority of breast malignancy cases $(55.7 \%)$ in T2 stage and 32\% moderately differentiated. [8] Khetarpal et al. found 76\% moderately differentiated, $18 \%$ well differentiated, and $6 \%$ poorly differentiated breast cases. [9] Dedavid et al. found $82 \%$ of colorectal cancers in stage T3/T4 and $61 \%$ belonging to well/moderately differentiated group. [10] Suchitra et al. found $40 \%$ oral malignancies in T4 stage [11].

We found significant association between MLR with tumor stage and tumor grade ( $\mathrm{p}$-value <
0.001), which was comparable to the study done by Saedi et al. [3].

A study done by Chen et al. on $\mathrm{HN}$ case reported that patient with higher MLR was shown to have poor survival. [4] LNR and number of positive lymph nodes are important prognostic factors with regard to overall survival for patients with nodepositive breast cancer, but LNR has a more correlated value in breast cancer subtypes [5]. Lee et al. in his published study declared that the MLR was a simple and reproducible prognostic factor that supplemented the limitation of the conventional $\mathrm{N}$ staging system and provided more accurate prognostic stratification in advanced gastric cancer [12]. Kwon et al. in his study showed that MLR is one of the main factors in predicting 5 years survivals of patients with gastric cancer [13]. Lymph node (LN) metastasis is one of the most important prognostic factors of gastric cancer [3]. Wright et al. found that higher MLR was associated with higher risk of death in GUT cases [14].

\section{CONCLUSIONS}

Previous studies did not define a specific cutoff value for MLR, but patients with a higher MLR were shown to have poor survival. In addition to the AJCC TNM classification system, LNR may be useful in stratifying risk in patients with primary organ malignancy. MLR is more valuable and reliable prognostic factor than total metastatic $\mathrm{LN}$ because it removes the effects of total number of resected LNs in determining patient prognosis. The routine inclusion of MLR in tumor reporting by pathologists may become a prognostic aid for clinicians along with TNM staging system. This can predict relapse and survival of cancer patients more closely.

\section{REFERENCES}

1. Kar, A.(2019). Prognostic value of lymph node ratio in cancer. Indian J Pathol Microbiol, 62:1978.

2. Sweety, S.V., Narayankar, A.S. (2019). Evaluation of lymph node ratio and morphologic patterns of nodal reactive hyperplasia in primary organ malignancy. Indian J Pathol Microbiol. 62: 21621.

3. Saedi, H.S., Fanipakdel, A., Shafaghi, A., Ghorbani, S. (2015). Evaluating the relation between patients-related factors and dissected lymph node ratio in gastric cancer. Indian J Cancer.

4. Chen, C.C., Lin, J.C., Chen, K.W. (2015). Lymph node ratio as a prognostic factor in head and neck cancer patients. Radiat Oncol, 10:181.

5. Liao, G.S., Chou, Y.C., Golshan, M., Hsu, H.M., Hong, Z.J., Yu, J.C.(2015). Prognostic value of the lymph node ratio in breast cancer subtypes, 210(4): 749-754.

6. Akagi, Y., Kansakar, R., \& Shirouz, K. (2012). The Prognostic Significance of Number of Lymph 
Node Metastasis in Colon Cancer-Based on Japanese Techniques of Resection and Handling of Resected Specimens. In Colorectal Cancer-From Prevention to Patient Care. IntechOpen.

7. Zeng, W.J, Hu, W.Q, Wang, L.W, Yan, S.G,. Li, J.D., Zhao, H.L.(2013). Lymph node ratio is a better prognosticator than lymph node status for gastric cancer: A retrospective study of 138 cases. Oncol Lett, 6:1693-700.

8. Titipungul, T., Intarawichian, P., Waraasawapati, S., Sangkhamanon, S., Chaisuriya, N., Pairojkul, C. (2015). Axillary lymph node ratio is an independent prognostic factor in Thai breast cancer patients. Asian Arch Pathol, 11:26-35.

9. Khetarpal, S., Mathur, S., Sethi, D., Sen, R. (2013). Immune hyperplasia patterns in lymph nodes draining breast cancer: A correlation with histomorphological parameters. Clin Cancer Investig J. 2:330-8.

10. Dedavid, e Silva, T.L., Damin, D.C. (2013). Lymph node ratio predicts tumor recurrence in stage III colon cancer. Rev Col Bras Cir; 40:46370 .

11. Suchitra, G., Puranik, R.S., Vanaki, S.S., Prasad, B.G., Malgaonkar, N.I. (2015). Immuno-reactivity of excised lymph nodes in neck dissections of squamous cell carcinomas of oral cavity. J Oral Maxillofac Pathol, 19:128-33.

12. Lee, S.Y., Hwang, I., Park, Y.S., Gardner, J., Ro, J.Y.(2010). Metastatic lymph node ratio in advanced gastric carcinoma: A better prognostic factor than number of metastatic lymph nodes? Int J Oncol, 36:1461-7.

13. Kwon, Y.M., Lim, H.T, Lee, K., Cho, B.L., Park, M.S., Son, K.Y. (2009). Factors associated with use of gastric cancer screening services in Korea. World J Gastroenterol, 15:3653-9.

14. Wright, J.L., Lin, D.W., Porter, M.P. (2008). The association between extent of lymphadenectomy and survival among patients with lymph node metastases undergoing radical cystectomy. Cancer, 112:2401-8. 